\title{
Improving the reporting and usability of research studies
}

\author{
Larissa Shamseer, MSc $\cdot$ James Galipeau, PhD • \\ Lucy Turner, MSc $\cdot$ David Moher, PhD
}

Received: 14 January 2013/ Accepted: 16 January 2013/Published online: 6 February 2013

(C) Canadian Anesthesiologists' Society 2013

Biomedical research and development is a multi-billion dollar industry that is failing to produce high-quality usable reports of research methods and findings. Poor reporting of research is serious, leading to a waste of investment in research, resources, and especially in data from patients participating in research who assume they are making useful contributions to health-care decision-making. Among the factors contributing to poorly reported studies is the lack of training, awareness, and knowledge about existing tools for those more directly involved in the publication process of research - authors, editors, and peer reviewers. Reporting guidelines are tools that have evolved from the early days of the original Consolidated Standards of Reporting Trials (CONSORT) Statement to over 200 guidelines and a formal network (EQUATOR) of guideline producers and users. Despite increasing development of different study designs and types of data, the value of reporting guidelines is still not being widely realized. Through support by journals publishing health research, reporting guidelines have been shown to have a positive influence on the completeness of reported research. Nevertheless, one of the fundamental challenges of reporting guidelines is ensuring their uptake and use at the optimal time and by relevant individuals in the research process. Additional resources would help improve the current dismal state of the reported medical literature. Two such examples include development of electronic tools to facilitate integration and ease of use of reporting guidelines

L. Shamseer, MSc $(\bowtie) \cdot$ J. Galipeau, PhD $\cdot$ L. Turner, MSc .

D. Moher, $\mathrm{PhD}$

Clinical Epidemiology Program, Ottawa Hospital Research Institute, Centre for Practice-Changing Research, 501 Smyth Rd, Box 201B, Ottawa, ON K1H 8L6, Canada

e-mail: 1shamseer@ohri.ca into manuscript writing and the peer review process as well as further training of all contributors to the problem. The purpose of this editorial is to highlight, for the benefit of readers and authors alike, several of the currently available resources to improve the reporting and usability of biomedical research.

\section{Poor reporting is a serious problem}

More than $\$ 200$ billion are spent globally on biomedical research every year, an investment that produces approximately one million publications. Despite such a large investment, over $85 \%$ of it is estimated to be lost in the form of preventable waste that accrues over four stages of the research process, one of which is the production of unusable and biased reports. ${ }^{1}$ Poorly reported studies fail to inform readers about the methods and findings of research in a clear and transparent manner, and they obscure the true representation of the methodological quality of a study, which may also be weak. Ambiguous reporting also makes a poorly described study nearly indistinguishable from a poorly designed and/or conducted study, creating difficulties in determining which evidence is of high enough quality and should contribute to healthcare decision-making. Evidence of this problem is increasingly accumulating. ${ }^{1-6}$

Poor reporting is unacceptable and unethical, particularly for patients participating in research who assume that all studies are published and reported in such a way that the findings can be synthesized with existing knowledge and used as part of healthcare decision-making. ${ }^{2,3}$ Furthermore, the public expects and assumes that research, particularly that which is publicly funded, is conducted and reported to the highest possible standards. Unfortunately, there are serious systemic problems regarding how research is 
reported, and despite the threat this poses on the integrity of our entire research enterprise, the problem has gone virtually ignored up to this point.

\section{Contributors to poorly reported research}

There are a number of different stakeholders contributing to poorly reported research. Scientists and health practitioners are the primary producers of health research and play a direct role in controlling the content of research reports. While many have training in research methods, they often have little or no exposure to the necessary skills to help them prepare complete, accurate, and transparent accounts of their methods and findings - reports that are "fit for purpose". ${ }^{4}$ Unfortunately, peer reviewers and editors also have little training on research reporting; no certification programs or degrees exist that allow a healthcare professional to train specifically to become a medical journal editor. ${ }^{5-7}$ Many editors report having informal or little or no training in editing skills ${ }^{8}$ and report being unfamiliar with available guidelines as well. ${ }^{9}$

\section{One solution to poor reporting}

One simple cost-effective solution - reporting guidelines emerged in the early $1990 \mathrm{~s}$ to help authors prepare their research for publication and to aid reviewers and editors in deciding what to publish. Many reporting guidelines provide checklists of an essential set of minimum items to be included in a study report. Checklists are powerful tools and, when adhered to, have been instrumental in transforming entire industries. ${ }^{10}$ When used in medical practice, they have been shown to reduce morbidity and mortality significantly while reducing healthcare costs. ${ }^{11,12}$ While medical checklists have the ability to reduce wasteful spending and improve efficiencies in patient care, reporting checklists have a similar potential to reduce waste in research by improving its completeness, accuracy, and clarity.

Nearly 20 years ago, a relatively small group of international scientists first met to develop a tool to improve the long-standing gap between what should be and what is contained in reports of randomized controlled trials (RCTs). ${ }^{13}$ Their intention was to produce a checklist that would facilitate the production of accurate and complete accounts of clinical trials and allow readers and reviewers to make judgements about their internal and external validity. Today, that initiative, the CONsolidated Standards Of Reported Trials (CONSORT) Statement, ${ }^{14}$ is regarded as one of the most important milestones in health research during the $20^{\text {th }}$ century. ${ }^{15}$ In its most up-to-date form,
CONSORT 2010 includes a 25-item checklist, a flow diagram to document the flow of participants throughout a trial, and a document with the rationale and guidance on how to report each checklist item. ${ }^{16}$ In the years since CONSORT was first published, over 200 other reporting guidelines (many also containing checklists) have emerged for reporting different study designs and types of data, along with an overarching initiative aimed at Enhancing the Quality and Transparency of Research - the EQUATOR Network (www.equator-network.org). According to metrics tracked by Google Scholar, CONSORT 2010 and a few other guidelines, namely, STROBE (Strengthening the Reporting of Observational Studies), ${ }^{17,18}$ STARD (Standards for Reporting Diagnostic accuracy studies), ${ }^{19,20}$ and PRISMA (Preferred Reporting Items for Systematic Reviews and Meta-analyses), ${ }^{21,22}$ are among the top-cited content in the journals that publish them, which, in-turn, are among the 100 top-cited journals in the world across the fields of health, engineering, and social sciences.

While reporting guidelines are an essential tool to help maintain the integrity of reporting, their integration into the publication process appears to be relatively minimal. ${ }^{23}$ Often, an author's first encounter with a reporting guideline, if at all, is when consulting a journal's " Instructions for Authors" while preparing their research manuscript for publication. There are multiple problems with this situation. First, journal instructions regarding reporting guidelines have been characterized as ambiguous and inconsistent, leaving authors confused as to what is expected of them. ${ }^{23}$ Second, poor, improper, incomplete, or non-use of reporting checklists by authors leaves the onus of identifying and addressing reporting problems on peer reviewers and editors, many of whom work on a volunteer basis and are primarily tasked with gauging the relevance and impact of a manuscript on their journal.

\section{Challenges of reporting guidelines}

While some guidelines have received widespread uptake, research is still not being reported in a manner that is entirely useful to readers. ${ }^{24}$ Several studies have attempted to gauge the impact of reporting guidelines; ${ }^{25-29}$ however, research on the effect of efforts to ensure adherence to reporting guidelines is missing from this body of evidence.

Endorsement of reporting guidelines by journals has been the main mechanism for their uptake and dissemination to target users, namely, authors and peer reviewers. Such endorsement is typically shown by a statement of support in a journal's "Instructions for Authors", encouraging or requiring authors to adhere to a relevant guideline checklist when submitting their manuscript. A simple endorsing statement, however, does not ensure use of 
reporting guidelines in the publication process. This is revealed in the findings of a recent systematic review assessing the effect of CONSORT endorsement on the completeness of trial reporting. While many CONSORT checklist items were better reported in trials published in endorsing journals, results of the review showed that they are still reported at exceedingly low levels. ${ }^{30}$ For instance, sequence generation and allocation concealment - the two most distinguishing features of RCTs - are completely reported less than $50 \%$ of the time, even among CONSORT-endorsing journals.

\section{The future of reporting guidelines}

Institutional involvement

The trajectory of manuscript publication often begins upon completion of a research project when an author starts drafting the manuscript for submission to a journal; it ends with the eventual publication of the manuscript after consideration by peer reviewers and editors. Currently, authors may not be aware of a particular reporting guideline prior to consulting a journal's "Instructions for Authors", and they may have difficulty modifying their drafted manuscript to ensure its adherence to the guideline. This highlights a need for awareness of guidelines earlier in the process, suggesting a more prominent role for institutions in the manuscript preparation process.

\section{Technological tools}

In interviews and surveys with editors and authors about their experience with reporting guidelines, both groups indicated the desire for a simpler way to complete the checklists and ensure compliance. ${ }^{\mathrm{A}}$ Several initiatives are underway, including the development of Web-based applications that will facilitate adherence to reporting guidelines by all contributors to the manuscript publication process.

A content management system that would streamline author adherence to reporting checklists is currently being developed. The system would allow reviewers to gauge more easily whether the study is adequately reported, and it would create a level playing field amongst all stakeholders by enabling the peer review process to take place interactively. One feature of the system would facilitate authors'

\footnotetext{
${ }^{\mathrm{A}}$ Moher D. Barriers and facilitators to CONSORT endorsement; 2011.
}

use of colour-coded semantic tagging. This would allow authors to tag sections of an uploaded manuscript that are pertinent to checklist items in a selected guideline, and it would make it easier for reviewers and editors to identify whether items are reported and to pinpoint where items are reported in the article. Eventually, we expect that such tagging would be accomplished using an automated process, such as data/text mining, that would also ensure compliance with checklist items and ease the workload of peer reviewers. Prototypes for emerging technologies are expected to be available later in 2013 .

\section{Improving consistency in journal endorsement statements}

Undoubtedly, professional communities want high-quality high-impact research to be published in their journals. Most immediately, it would be beneficial for journals to adopt a uniform message regarding their expectations about authors' (and peer reviewers') use of reporting guidelines. For instance, the following language is suggested for endorsement of CONSORT 2010 in the "Instructions for Authors" section, "(This journal) requires a completed CONSORT 2010 checklist and flow diagram as a condition of submission when reporting the results of a randomized trial. Templates for these can be found here or on the CONSORT Web site (www.consort-statement.com), which also describes several CONSORT checklist extensions for different designs and types of data beyond two-group parallel trials. Authors should ensure, at minimum, that each item on the checklist is addressed in the content of the manuscript. Meeting these basic reporting requirements will greatly improve the value of the trial report and may enhance its chances for eventual publication." The Canadian Journal of Anesthesia, a strong advocate of reporting guidelines, comes close to this language.

There is plenty of work to be done if there is to be meaningful change in research reporting. New ideas must be put forth and explored, such as the promotion and integration of reporting guidelines and other resources earlier in the research process. Taking account of the criteria for complete and transparent reporting at the start of a research project instead of near the end could create major improvements not only in the reporting of research but also in its design. ${ }^{31}$ Similarly, leveraging cutting-edge technology can lead to greater efficiency in the writing, editing, and peer review processes. Nevertheless, the most critical factor in improving the integrity of research reporting is for authors, editors, and peer reviewers to recognize the seriousness of the problem and the danger it poses on our scientific enterprise and the health and trust of those who depend on it. 


\section{L'amélioration de la présentation et de l'exploitation des résultats de recherche}

Le secteur recherche et développement dans le domaine biomédical est une affaire de plusieurs milliards de dollars; toutefois, ce secteur peine à produire des comptes rendus des méthodes et des résultats de recherche qui soient de bonne qualité et utilisables. La qualité médiocre de la présentation des résultats de recherche est un problème grave, qui entraîne du gaspillage en matière d'investissements dans la recherche, de ressources, et tout particulièrement de données des patients qui participent aux études - lesquels pensent qu'ils contribuent de façon utile à la prise de décision en soins de santé. Parmi les facteurs conduisant à cette mauvaise présentation des résultats, citons le manque de formation, de sensibilisation et de connaissances concernant les outils existants destinés aux personnes directement impliquées dans le processus de publication de la recherche - les auteurs, les rédacteurs et les lecteurs experts. Les directives de présentation sont des outils qui ont bien évolué depuis les débuts du premier Énoncé CONSORT (Consolidated Standards of Reporting Trials). Aujourd'hui, il existe plus de 200 directives ainsi qu'un réseau formel (EQUATOR) de rédacteurs et d'utilisateurs de directives. Malgré des mises au point pour un nombre de plus en plus abondant de plans d'études et de types de données, les directives de présentation n'occupent toujours pas la place qu'elles méritent. Grâce au soutien des revues de recherche en santé, il a été démontré que les directives de présentation exerçaient une influence positive sur l'exhaustivité des données rapportées. Cependant, l'un des défis principaux des directives de présentation consiste à garantir leur adoption et leur utilisation au moment opportun et par les bonnes personnes dans le processus de recherche. Des ressources supplémentaires, telles que la mise au point d'outils électroniques pour faciliter l'intégration et la facilité d'utilisation des directives de présentation dans les processus de rédaction d'un manuscrit et de son évaluation par les pairs, ainsi qu'une formation plus poussée de toutes les personnes contribuant au problème - voilà ce qui aiderait à améliorer l'état déplorable actuel de la présentation des données en littérature médicale. L'objectif de cet éditorial est de présenter, au profit des lecteurs et des auteurs, plusieurs des ressources actuellement à leur disposition pour améliorer la présentation et l'exploitation des données de recherche biomédicale.

\section{Une présentation boiteuse est un problème grave}

Plus de 200 milliards \$ sont dépensés chaque année dans le monde pour la recherche biomédicale, et cet investissement génère environ un million de publications. Malgré l'ampleur de cet investissement, on estime que plus de $85 \%$ de ce montant se perd en prenant la forme de gaspillage évitable, lequel s'étend sur quatre étapes $\mathrm{du}$ processus de recherche. La production d'articles inutilisables et biaisés est l'une d'elles. ${ }^{1}$ Les études mal présentées ne réussissent pas à informer, de façon claire et transparente, le lecteur quant aux méthodes et aux résultats d'une recherche. Ils occultent la véritable représentation de la qualité méthodologique d'une étude, qui pourrait elle aussi être médiocre. Lorsqu'une étude mal décrite est, de surcroît, rapportée de façon ambiguë, cela la rend quasiment indifférenciable d'une étude mal conçue ou mal menée, ce qui crée des difficultés lorsqu'on tente de déterminer quelles données probantes sont de qualité suffisante et devraient contribuer à la prise de décision en soins de santé. Les données probantes soulignant ce problème s'accumulent de plus en plus. ${ }^{1-6}$

Une mauvaise présentation des résultats d'une recherche est à la fois inacceptable et contraire à l'éthique, particulièrement envers les patients qui participent à la recherche et qui présument que toutes les études sont publiées et présentées de façon à ce que les résultats puissent être intégrés aux connaissances existantes et utilisés dans le cadre de la prise de décision en soins de santé. ${ }^{2,3}$ En outre, le public escompte et présume que la recherche, particulièrement celle financée par les deniers publics, est menée et présentée selon les normes les plus élevées qui soient. Malheureusement, il existe des problèmes graves et systémiques dans la façon dont la recherche est présentée. Malgré la menace que cela pose à l'intégrité de notre système de recherche dans son intégralité, le problème est passé littéralement inaperçu à ce jour.

\section{Les personnes contribuant à la mauvaise présentation de la recherche}

Plusieurs acteurs jouent un rôle dans la présentation médiocre des résultats de recherche. Les scientifiques et les professionnels de la santé génèrent la majorité des résultats de recherche en santé et jouent un rôle direct dans le contrôle du contenu des comptes rendus de recherche. Alors que bon nombre d'entre eux ont reçu une formation concernant les diverses méthodes de recherche, ils ne sont que peu ou prou exposés aux compétences nécessaires qui 
les aideraient à préparer des comptes rendus complets, précis et transparents de leurs méthodes et de leurs résultats - des comptes rendus « fonctionnels ». ${ }^{4}$ Malheureusement, les lecteurs experts et les rédacteurs sont peu formés quant à la présentation des résultats de recherche; il n'existe ni programme, ni diplôme d'accréditation permettant à un professionnel de la santé de se former spécifiquement à devenir un rédacteur de revue médicale. ${ }^{5-7}$ Plusieurs rédacteurs rapportent n'avoir qu'une formation informelle - voire aucune formation - en matière de compétences de rédaction et de révision et avouent ne pas bien connaître les directives disponibles non plus. ${ }^{9}$

\section{Une solution à la présentation médiocre de résultats}

Une solution simple et rentable - les directives de présentation - a vu le jour au début des années 1990 afin de guider les auteurs dans la préparation leurs résultats pour une éventuelle publication et d'aider les évaluateurs et les rédacteurs à décider que publier. Nombre de directives de présentation proposent des listes de contrôle comprenant un ensemble minimal d'éléments à inclure dans un compte rendu d'étude. Les listes de contrôle sont des outils puissants et, lorsqu'elles ont été respectées, ont joué un rôle essentiel en transformant des secteurs entiers. ${ }^{10}$ Il a été démontré que lorsqu'elles sont utilisées dans la pratique médicale, les listes de contrôle réduisaient de façon significative la morbidité et la mortalité tout en réduisant les coûts des soins de santé. ${ }^{11,12}$ Alors que les listes de contrôle en médecine peuvent réduire les dépenses inutiles et améliorer l'efficience dans les soins aux patients, les listes de contrôle concernant la présentation des résultats de recherche possèdent un potentiel semblable pour réduire le gaspillage en recherche en la rendant plus complète, plus exacte et plus claire.

Il y a près de 20 ans, un groupe relativement restreint de scientifiques internationaux se sont rencontrés pour mettre au point un outil visant à réduire le vieux fossé existant entre ce qui devrait être présenté et ce qui l'est effectivement dans les comptes rendus d'études randomisées contrôlées (ERC). ${ }^{13}$ Leur intention était de mettre au point une liste de contrôle afin de faciliter la production de comptes rendus exacts et complets des études cliniques, dans le but de permettre l'évaluation de leur validité interne et externe par les lecteurs experts ou non. Aujourd'hui cette initiative, l'Énoncé CONSORT (CONsolidated Standards Of Reported Trials), ${ }^{14}$ est considérée comme l'un des jalons les plus importants en recherche en santé du $20^{\mathrm{e}}$ siècle. ${ }^{15}$ Sous sa forme la plus récente, l'Énoncé CONSORT 2010 comprend une liste de contrôle de 25 éléments, un organigramme afin de documenter le parcours des participants au projet tout au long de l'étude, ainsi qu'un document donnant des raisons et des conseils pour respecter chaque élément de la liste de contrôle. ${ }^{16}$ Depuis la première édition de l'Énoncé CONSORT, plus de 200 autres directives de présentation (dont plusieurs incluent également des listes de contrôle) ont vu le jour pour améliorer la présentation de différents types d'études et de données; en outre, une initiative globale visant à améliorer la qualité et la transparence de la recherche, le Réseau EQUATOR (Enhancing the Quality and Transparency of Research - www.equator-network.org ), a vu le jour. Selon des mesures effectuées à l'aide de Google Scholar, l'Énoncé CONSORT 2010 et quelques autres directives, notamment STROBE (Strengthening the Reporting of Observational Studies), ${ }^{17,18}$ STARD (Standards for Reporting Diagnostic accuracy studies) $)^{19,20}$ et PRISMA (Preferred Reporting Items for Systematic Reviews and Meta-analyses), ${ }^{21,22}$ comptent parmi les articles les plus cités des revues qui les publient; ces revues sont, à leur tour, parmi les 100 revues les plus citées au monde dans les domaines de la santé, de l'ingénierie et des sciences sociales.

Bien que les directives de présentation constituent un outil essentiel pour aider à conserver l'intégrité des communications en recherche, leur intégration dans le processus de publication semble être relativement restreint. ${ }^{23}$ Souvent, un auteur prend conscience pour la première fois de l'existence d'une directive de présentation - s'il en prend conscience - lorsqu'il consulte les «Directives aux auteurs» d'une revue durant la préparation de son manuscrit de recherche pour publication. Cet état de fait comprend de nombreux problèmes. En premier lieu, les instructions des revues concernant les directives de présentation ont été décrites comme étant ambiguës et incohérentes, ce qui rend les auteurs perplexes quant à ce qui est attendu d'eux. ${ }^{23}$ Deuxièmement, l'utilisation médiocre, incorrecte et incomplète, voire la non-utilisation, des listes de contrôle de présentation par les auteurs relègue la responsabilité d'identifier et de traiter des problèmes de présentation aux lecteurs experts et aux rédacteurs, dont bon nombre travaillent de façon bénévole et dont le rôle principal est d'évaluer la pertinence d'un article et son impact sur leur revue.

\section{Les défis des directives de présentation}

Alors que certaines directives ont été adoptées de façon généralisée, les recherches ne sont toujours pas présentées d'une façon complètement utile aux lecteurs. ${ }^{24}$ Plusieurs études ont tenté d'évaluer l'impact des directives de présentation. ${ }^{25-29}$ Toutefois, il manque à ce corpus de données probantes des recherches concernant l'effet des 
efforts faits pour garantir le respect des directives de présentation.

L'appui aux directives de présentation par les revues a été le mécanisme principal de leur adoption par et de leur diffusion aux utilisateurs cibles - c'est-à-dire les auteurs et les lecteurs experts. Un tel appui est en général démontré par une déclaration de soutien dans les «Directives aux auteurs » d'une revue, qui encourage ou oblige les auteurs à respecter la liste de contrôle d'une directive pertinente lors de la soumission de leur manuscrit. Mais une simple déclaration de soutien ne garantit toutefois pas l'utilisation d'une directive de présentation dans le processus de publication. Les résultats d'une revue méthodique récente évaluant l'effet de l'adoption de l'Énoncé CONSORT sur l'exhaustivité dans la présentation des résultats des études a mis en exergue cet écueil en montrant que, bien que de nombreux items de la liste de contrôle CONSORT étaient mieux présentés dans les études publiées dans des revues ayant appuyé l'énoncé, ces items étaient néanmoins toujours rapportés à des niveaux excessivement bas. ${ }^{30}$ Par exemple, le processus de génération de séquence et de dissimulation de l'attribution, les deux éléments les plus distinctifs d'une ERC - sont complètement décrits dans moins de $50 \%$ des cas et ce, même dans les revues ayant adopté l'Énoncé CONSORT.

\section{L'avenir des directives de présentation}

Implication des institutions

La trajectoire de publication d'un manuscrit commence souvent à la fin d'un projet de recherche, lorsqu'un auteur commence à rédiger un manuscrit qu'il a l'intention de soumettre à une revue; elle se termine avec la publication éventuelle d'un article après son évaluation par des lecteurs experts et des rédacteurs. À l'heure actuelle, les auteurs pourraient ne pas connaître une directive de présentation spécifique avant de consulter les «Directives aux auteurs » d'une revue, ce qui leur complique la tâche de modifier leur ébauche de manuscrit pour garantir qu'il respecte une directive de présentation donnée. Ceci souligne le besoin de connaître les directives plus tôt dans le processus, ce qui impliquerait un engagement plus prépondérant des institutions dans le processus de préparation des manuscrits.

\section{Les outils technologiques}

Dans des entrevues et des sondages réalisés auprès de rédacteurs et d'auteurs concernant leur expérience avec les directives de présentation, les deux groupes ont fait part de leur désir de disposer d'une façon plus simple de compléter les listes de contrôle et de garantir leur respect. ${ }^{\mathrm{A}}$ Plusieurs initiatives sont en cours, notamment la mise au point d'applications basées sur Internet qui faciliteront le respect des directives de présentation par tous les intervenants dans le processus de publication d'un manuscrit.

Un système de gestion du contenu est actuellement en cours de développement. Ce système rationnaliserait le respect par les auteurs des listes de contrôle de présentation, permettant ainsi aux lecteurs experts d'évaluer plus facilement la présentation conforme d'une étude. Ce système créerait des règles équitables pour tous les intervenants en permettant au processus de révision par les pairs de se dérouler de façon interactive. L'une des propriétés de ce système faciliterait l'utilisation, par les auteurs, d'étiquetage sémantique par code de couleurs, afin d'étiqueter certaines sections d'un manuscrit téléchargé comme respectant certains éléments de la liste de contrôle d'une directive en particulier. Ainsi, il serait plus facile pour les lecteurs experts et les rédacteurs de trouver où et si les éléments d'une liste de contrôle sont décrits. Nous anticipons qu'un jour un tel étiquetage se fera via un processus automatisé tel que l'exploration de données ou de texte, ce qui permettrait en outre de garantir le respect des éléments d'une liste de contrôle, allégeant ainsi la charge de travail des lecteurs experts. Des prototypes de technologies émergentes devraient être disponibles plus tard en 2013.

\section{L'amélioration de la cohérence dans les énoncés d'appui des revues}

Il ne fait aucun doute que les revues veulent que les études qu'elles publient soient de qualité élevée et aient un fort impact. Dans l'immédiat, il serait utile que les revues adoptent un message uniforme concernant leurs attentes quant à l'utilisation des directives de présentation par les auteurs (et par les lecteurs experts). Par exemple, concernant le soutien de l'Énoncé CONSORT 2010, la terminologie suivante est suggérée pour leur section « Directives aux auteurs » : « [cette revue] exige une liste de contrôle et un organigramme CONSORT 2010 complétés en tant que condition de soumission lors de la présentation des résultats d'une étude randomisée. Des modèles sont disponibles ici ou sur le site Internet de CONSORT [www. consort-statement.com], lequel décrit également plusieurs extensions de la liste de contrôle CONSORT concernant diverses méthodologies et différents types de données pour les études autres que celles évaluant deux groupes en parallèle. Vous devez vous assurer que votre article

\footnotetext{
A Moder D, Les obstacles et les facilitateurs à l'adoption de CONSORT, 2001.
} 
présente, au minimum, du contenu correspondant à chaque élément de la liste de contrôle. Le respect de ces exigences de base améliorera grandement la valeur de votre compte rendu d'étude et pourrait améliorer ses chances de publication éventuelle. » Le Journal canadien d'anesthésie, un fervent défenseur des directives de présentation, utilise une terminologie proche de cet exemple.

Le travail est de taille si l'on souhaite accomplir des changements significatifs dans la présentation des résultats de recherche. Il faut trouver et explorer de nouvelles idées, comme par exemple la promotion et l'intégration des directives de présentation et d'autres ressources plus tôt dans le processus de recherche. Le fait de tenir compte des critères nécessaires à la présentation complète et transparente de la recherche dès les débuts d'un projet de recherche plutôt que vers la fin pourrait apporter des améliorations considérables non seulement à la façon dont les résultats sont rapportés, mais aussi à la façon dont la recherche est conçue. ${ }^{31}$ De même, si l'on parvenait à tirer profit des technologies de pointe, ceci entraînerait une meilleure efficacité dans les processus de rédaction, de correction et d'évaluation par les pairs. Toutefois, le facteur crucial pour améliorer l'intégrité de la présentation des résultats de recherche est la prise de conscience, par ceux qui génèrent des connaissances, de la gravité du problème et des dangers qu'il pose à notre projet scientifique, ainsi qu'à la santé et à la confiance de ceux qui en dépendent.

Competing interests Dr. David Moher is an author of the CONSORT and PRISMA Statements. Ms. Shamseer works as a Research Coordinator for the CONSORT group; her salary is paid in part by a grant from the Medical Research Council, UK.

Funding Sources None.

\section{References}

1. Chalmers I, Glasziou P. Avoidable waste in the production and reporting of research evidence. Obstet Gynecol 2009; 114: 1341-5.

2. Moher D. Reporting research results: a moral obligation for all researchers. Can J Anesth 2007; 54: 331-5.

3. Groves T. Enhancing the quality and transparency of health research. BMJ 2008; 337: a718.

4. Simera I, Altman DG. Writing a research article that is "fit for purpose": EQUATOR Network and reporting guidelines. Evid Based Med 2009; 14: 132-4.

5. Kassirer JP. msJAMA: Why be a medical editor? JAMA 2001; 285: 2253.

6. Wong VS. The training of physician-editors: a call for a more formal approach. Sci Ed 2008; 31: 75-7.

7. Wong VS, Callaham ML. Medical journal editors lacked familiarity with scientific publication issues despite training and regular exposure. J Clin Epidemiol 2012; 65: 247-52.

8. Garrow J, Butterfield M, Marshall J, Williamson A. The reported training and experience of editors in chief of specialist clinical medical journals. JAMA 1998; 280: 286-7.
9. Wager E, Fiack S, Graf C, Robinson A, Rowlands I. Science journal editors' views on publication ethics: results of an international survey. J Med Ethics 2009; 35: 348-53.

10. Gawande A. The Checklist Manifesto: How to Get Things Right. NY: Metropolitan Books; 2010.

11. Pronovost $P$, Needham $D$, Berenholtz $S$, et al. An intervention to decrease catheter-related bloodstream infections in the ICU. N Engl J Med 2006; 26: 2725-32.

12. Haynes AB, Weiser TG, Berry WR, et al. A surgical safety checklist to reduce morbidity and mortality in a global population. N Engl J Med 2009; 360: 491-9.

13. Begg C, Cho M, Eastwood $S$, et al. Improving the quality of reporting of randomized controlled trials. The CONSORT statement. JAMA 1996; 276: 637-9.

14. Schulz KF, Altman DG, Moher D; CONSORT Group. CONSORT. statement: updated guidelines for reporting parallel group randomised trials. Ann Intern Med 2010; 2010(152): 726-32.

15. Gabriel SE, Normand ST. Getting the methods right - the foundation of patient-centered outcomes research. N Engl J Med 2012; 367: 787-90.

16. Moher D, Hopewell S, Schulz KF, et al. CONSORT 2010 explanation and elaboration: updated guidelines for reporting parallel group randomised trials. BMJ 2010; 340: c869.

17. von Elm E, Altman DG, Egger $M$, et al. Strengthening the Reporting of Observational Studies in Epidemiology (STROBE) statement: guidelines for reporting observational studies. BMJ 2007; 335: 806-8.

18. Vandenbroucke JP, von Elm E, Altman DG, et al. Strengthening the Reporting of Observational Studies in Epidemiology (STROBE): explanation and elaboration. PLoS Med 2007; 4: e297.

19. Bossuyt PM, Reitsma JB, Bruns DE, et al. Towards complete and accurate reporting of studies of diagnostic accuracy: the STARD initiative. Standards for Reporting of Diagnostic Accuracy. Clin Chem 2003; 49: 1-6.

20. Bossuyt PM, Reitsma JB, Bruns DE, et al. The STARD statement for reporting studies of diagnostic accuracy: explanation and elaboration. Clin Chem 2003; 49: 7-18.

21. Moher D, Liberati A, Tetzlaff J. PRISMA Group. Preferred reporting items for systematic reviews and meta-analyses: the PRISMA statement. BMJ 2009; 339: b2535.

22. Liberati A, Altman DG, Tetzlaff $J$, et al. The PRISMA statement for reporting systematic reviews and meta-analyses of studies that evaluate health care interventions: explanation and elaboration. PLoS Med 2009; 6: e1000100.

23. Hopewell S, Altman DG, Moher D, Schulz KF. Endorsement of the CONSORT statement by high impact factor medical journals: a survey of journal editors and journal 'Instructions to Authors'. Trials 2008; 9: 20.

24. Glasziou P, Meats E, Heneghan C, Shepperd S. What is missing from descriptions of treatment in trials and reviews? BMJ 2008; 336: 1472-4.

25. Smidt $N$, Rutjes $A W$, van der Windt $D$, et al. The quality of diagnostic accuracy studies since the STARD statement: has it improved? Neurology 2006; 67: 792-7.

26. Areia $M$, Soares $M$, Dinis-Ribeiro $M$. Quality reporting of endoscopic diagnostic studies in gastrointestinal journals: where do we stand on the use of the STARD and CONSORT statements? Endoscopy 2010; 42: 138-47.

27. Hammerschlag $R$, Milley $R$, Colbert A, et al. Randomized controlled trials of acupuncture (1997-2007): an assessment of reporting quality with a CONSORT-and STRICTA-based instrument. Evid Based Complement Alternat Med 2011; DOI: 10.1155/2011/183910.

28. Haidich AB, Birtsou C, Dardavessis T, Tirodimos I, Arvanitidou $M$. The quality of safety reporting in trials is still suboptimal: 
survey of major general medical journals. J Clin Epidemiol 2011; 64: $124-35$

29. Agha R, Cooper D, Muir G. The reporting quality of randomised controlled trials in surgery: a systematic review. Int J Surg 2007; 5: 413-22.

30. Turner L, Shamseer L, Altman DG, et al. Consolidated standards of reporting trials (CONSORT) and the completeness of reporting of randomised controlled trials (RCTs) published in medical journals. Cochrane Database Syst Rev 2012; 11: MR000030.

31. Williams HC. Cars, CONSORT 2010, and clinical practice. Trials 2010; 11: 33 . 